\section{PWE-203 CAPSULE ENDOSCOPY: A DISTRICT GENERAL HOSPITAL EXPERIENCE IN A MULTIETHNIC COMMUNITY}

doi:10.1136/gutjnl-2012-302514d.203

S Subramaniam, ${ }^{*}$ A Yusuf, S Shariq. Department of Gastroenterology, Ealing Hospital, London, UK

Introduction Capsule endoscopy (CE) is a useful tool to assess the small bowel (SB). The 2008 British Society of Gastroenterology (BSG) guidelines detailed the use of CE in occult gastrointestinal bleeding (OGIB) and suspected SB Crohn's disease (CD). ${ }^{1}$ At present, $\mathrm{CE}$ is not available in all district general hospitals (DGH). Our aim was to ascertain the indications for referral and evaluate the diagnostic yield of this test in a multiracial North West London DGH.

Methods We conducted a retrospective analysis of all CE undertaken from April 2009 to December 2011. 136 patients [60 male (44.1\%) and 76 female (55.9\%); mean age 53.1] were included in this study. Data were collected on patient demographics, previous diagnostic investigations, indications and findings of $\mathrm{CE}$.

Results See Abstract PWE-203 table 1 below. Patients referred for OGIB had negative bidirectional endoscopies. Patients with abdominal pain had imaging to exclude strictures. There were no cases of capsule retention. CE in OGIB revealed the main diagnoses were SB telangiectasia $(9 / 68 ; 13.2 \%)$, non-specific SB inflammation and ulceration $(9 / 68 ; 13.2 \%)$ and erosive gastritis $(7 / 68 ; 10.3 \%)$. Two cases each of portal hypertensive gastropathy, CD, coeliac disease and polypoid lesions were diagnosed (total 11.8\%) and one case of angiodysplasia and lipoma (2/68; $3 \%)$. Suboptimal views were obtained in 8/68 (11.8\%) while CE was normal in 25 patients (36.8\%). In the suspected CD group, 14 patients (14/57; $24.6 \%$ ) were diagnosed with CD (at least $3 \mathrm{SB}$ apthous ulcers and erosions). Nonspecific inflammation $(5 / 57 ; 8.8 \%)$ and erosive gastritis $(5 / 57 ; 8.8 \%)$ were also found. Suboptimal views affected five cases and 25 patients (43.9\%) had normal CE. One case each of coeliac disease, radiation enteritis and intestinal lymphangiectasia were also diagnosed. One case of SB lymphoma was diagnosed as a complication of coeliac disease. Subgroup analysis according to ethnicity in suspected CD revealed 29 Caucasian (50.9\%), 26 Asian (45.6\%) and $2(3.5 \%)$ patients of African descent. CD was diagnosed in 8 Caucasians and 6 Asians.

Abstract PWE-203 Table 1 Indications for referral

\begin{tabular}{ll}
\hline Indications & Number of patients (\%) \\
\hline Suspected SB CD & $57(41.9 \%)$ \\
OGIB & \\
$\quad$ Overt GI bleeding & $52(38.2 \%)$ \\
$\quad$ Iron deficiency anaemia & $16(11.8 \%)$ \\
Coeliac disease complications & $7(5.2 \%)$ \\
Suspected SB tumour & $3(2.2 \%)$ \\
Polyposis syndromes & $1(0.7 \%)$ \\
Total & 136 \\
\hline
\end{tabular}

Conclusion In our cohort, diagnostic yield for CD was less than other studies $(24.56 \%$ vs $43-71 \%) .{ }^{1}$ This may reflect the prevalence of $\mathrm{CD}$ in an Asian minority area. ${ }^{2} \mathrm{SB}$ telangiectasia and ulceration were the main sources of OGIB. CE is a safe and useful technique to evaluate the SB and we recommend its use in the DGH setting.

Competing interests None declared.

\section{REFERENCES}

1. Sidhu R, Sanders DS, Morris AJ, et al. Guidelines on small bowel enteroscopy and capsule endoscopy in adults. Gut 2008;57:125-36.

2. Yang SK, Loftus EV, Sandborn WJ. Epidemiology of IBD in Asia. Infl Bow Dis 2001; 7:260-70.

\section{PWE-204 DOES CHOLANGIOSCOPY IMPROVE THE DIAGNOSIS OF "INDETERMINATE" BILIARY STRICTURES?}

doi:10.1136/gutjnl-2012-302514d.204

${ }^{1,2} \mathrm{~S}$ Menon, ${ }^{2} \mathrm{~V}$ Lehkaraju, ${ }^{2} \mathrm{R}$ Sturgess. ${ }^{1}$ Department of Gastroenterology, Royal Wolverhampton Hospitals NHS Trust, Wolverhampton, UK; ${ }^{2}$ Department of Gastroenterology, University Hospital Aintree, Liverpool, UK

Introduction The precise assessment of a biliary stricture can be challenging. Multi-modal imaging with CT/MR and EUS can generally determine the nature of a biliary stricture and EUS in particular, can offer histological diagnosis. However, ERCP is often necessary for diagnostic evaluation and management of biliary strictures and biliary cytology performed at ERCP has a diagnostic yield of only $10-40 \%$. Assessment of focal intrahepatic strictures can thus, be even more difficult. Cholangioscopy offers the advantage of tissue sampling under direct vision, thereby enhancing diagnostic precision. The aim of the study was to review our experience of cholangioscopy in the assessment of biliary strictures Methods A review of a prospectively maintained database of all procedures involving per oral single operator cholangioscopy at our institution (June 2008-2011), was performed. "Indeterminate" strictures were defined as biliary strictures referred for diagnostic evaluation with previous negative cytology. Other biliary strictures were referred for evaluation after negative EUS-FNA, or directly based on radiological abnormalities. Follow-up data were acquired through case note review and phone calls to referring hospitals Summary statistics were applied.

Results 72 patients underwent cholangioscopy for "indeterminate strictures" during the study period (42 males, mean age 64 years (SD 11)). These patients had a total of 228 investigations performed as part of their evaluation (mean 3 investigations/person). 39 (54\%) had a previous ERCP (range $1-6$ procedures per person). At cholangioscopy, adequate mucosal visualisation was achieved in 64 $(89 \%)$ and direct biopsy sampling was carried out in $36(50 \%)$ patients. 35 patients needed prior sphincterotomy. Tissue diagnosis was achieved in 54 (75\%) patients (15 cholangiocarcinoma, 5 PSCrelated strictures, 12 ischaemic/inflammatory, 1 biliary leiomyoma and 21 normal tissue). $6(10 \%)$ had complications related to the procedure (5 cholangitis, 1 post-sphincterotomy bleed). On followup (mean 10 months (range $2-24$ months), four patients underwent surgery (2 cholangiocarcinoma, 2 ischaemic/inflammatory strictures), 13 had endoscopic palliation for cholangiocarcinoma, of which seven died (mean 9 months).

Conclusion Cholangioscopy is valuable tool in the diagnostic evaluation of biliary strictures.

Competing interests None declared.

\section{PWE-205 PROPOFOL-BALANCED ANAESTHESIA FOR SINGLE BALLOON ENTEROSCOPY: A COMPARISON BETWEEN ANTEGRADE AND RETROGRADE INTUBATION}

doi:10.1136/gutjnl-2012-302514d.205

S Amornyotin,* W Chalayonnawin, S Kongphlay. Department of Anesthesiology, Faculty of Medicine Siriraj Hospital, Mahidol University, Bangkok, Thailand

Introduction Single balloon enteroscopy (SBE) procedure is a procedure for diagnosis and treatment in patients with abnormality of gastrointestinal tract especially for small bowel. It commonly performed either anterograde or retrograde intubation. The aim of the study is to compare and evaluate the success rate of propofolbalanced anaesthesia (PBA) for the SBE procedure between antegrade and retrograde intubation.

Methods We undertook a retrospective review of the sedation service records of patients who underwent SBE by using PBA technique. All procedures were classified into two groups according to route of the 